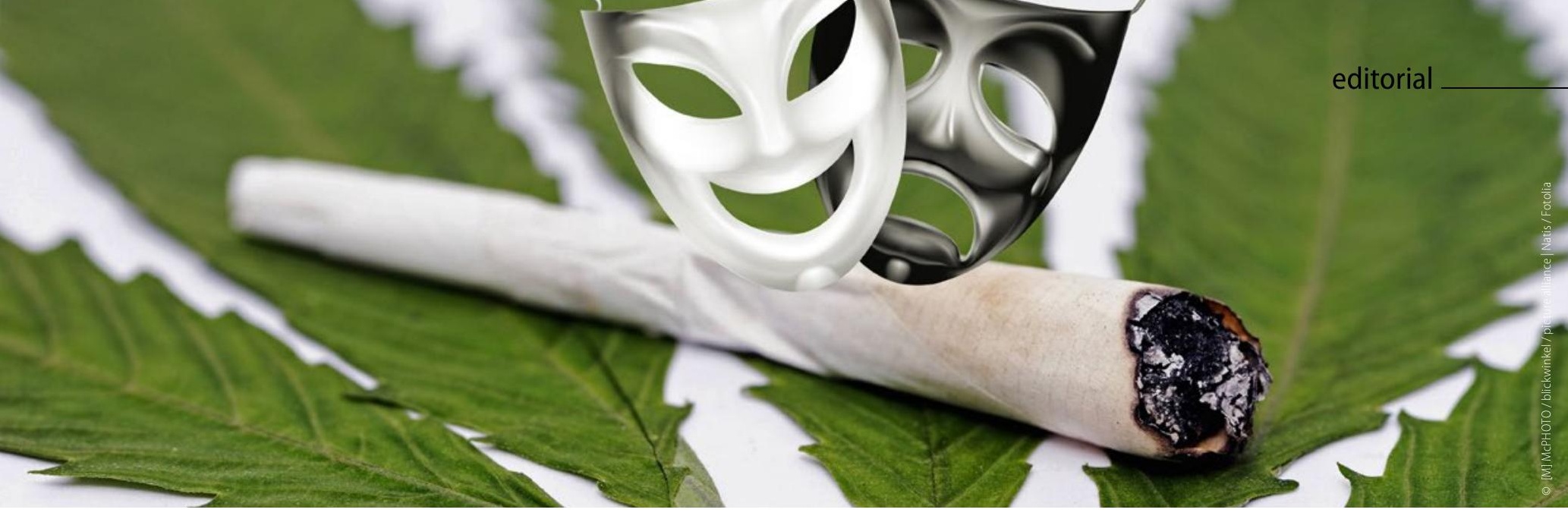

\title{
Cannabis gefährdet auch die Lunge!
}

Cannabis ist die in Deutschland am weitesten verbreitete illegale Droge, die von ca. 600.000 z.T. abhängigen Erwachsenen regelmäßig konsumiert wird. Erschreckend ist, dass sich der Konsum bei 12bis 17-Jährigen von 2011-2014 von 2,8 auf 6,4\%, der regelmäßige Konsums von 0,2 auf 1,5\% steigerte.

Aktuell wird in der Politik und der Öffentlichkeit eine intensive Debatte über den künftigen Umgang mit Cannabinoiden geführt, nachdem der Konsum von Cannabis in einigen Ländern freigegeben wurde. Allerdings müssen in diese Debatte unbedingt auch gesundheitliche Aspekte einfließen, da der Konsum nicht verharmlost werden darf und die Datenlage zu vermeintlich positiven Effekten gut beleuchtet werden muss. Die Deutsche Gesellschaft für Pneumologie und Beatmungsmedizin (DGP) hat daher mit Sucht- und Arbeitsmedizinern, Kardiologen sowie Kinderund Jugendärzten ein Positionspapier zu Cannabis erstellt [1].

\section{Gefahr steigt mit der Lebenszeitdosis}

Auf Basis der aktuellen Evidenz sind darin die Gesundheitsrisiken von Cannabis aufgeführt. Neben den psychischen und neurologischen werden in dem Positionspapier besonders die pneumologischen und kardialen Nebenwirkungen diskutiert. Als so gut wie gesichert gilt die Induktion einer chronischen Bronchitis, auch wenn der Kofaktor des gleichzeitigen Zigarettenrauchens abgezogen wird. Assoziationen mit anderen Krankheiten wie Lungenemphysem, Lungenkrebs und Pneumonien sind wahrscheinlich, aber nur unzureichend belegt. Auch kardiovaskuläre Erkrankungen wie das akute Koronarsyndrom, periphere Gefäßerkrankungen und zerebrale Komplikationen sind in Zusammenhang mit einem Cannabiskonsum beschrieben.

Allerdings reicht die Datenlage aus vielen Gründen oft für eindeutige Aussagen nicht aus. Vor allem kann in den meisten Studien nicht oder nur unzureichend zwischen den sich überlappenden Effekten des Tabakrauchens und des Cannabiskonsums differenziert werden. Empirisch ist aber sehr gut belegt, dass ein früh im Leben beginnender, hochdosierter, langjähriger und regelmäBiger Cannabisgebrauch das Risiko für verschiedene Störungen der psychischen und körperlichen Gesundheit und der altersgerechten Entwicklung erhöht. Daher gibt vor allem der Cannabiskonsum bei Kindern und Jugendlichen Anlass zur Sorge. Im Hinblick auf den oft diskutierten medizinischen Nutzen von Cannabinoiden ist die wissenschaftliche Datenlage nur gering und eine systematische Forschung guter Qualität liegt kaum vor. Zusammenfassend stimmen die an dem Positionspapier beteiligten Gesellschaften darin überein, dass noch viele Aspekte hinsichtlich der gesundheitlichen Folgen von Cannabis offen sind und möglichst in kontrollierten Studien geklärt werden sollten.

\section{Literatur:}

1. Cannabis - Positionspapier der Deutschen Gesellschaft für Pneumologie und Beatmungsmedizin e.V. (DGP) in Zusammenarbeit mit DGAUM, DGS, DG-Sucht, DGKPJ, GPP, DGKJ, BVKJ, BdP, DGK, Herzstiftung. Kreuter M, Nowak D, Rüther T, Hoch E, Thomasius R, Vogelberg C, Brockstedt M, Hellmann A, Gohlke H, Jany B, Loddenkemper R. Pneumologie. 2016 Feb;70(2):87-97. im Internet unter: http://pneumologie.de/111.0.html

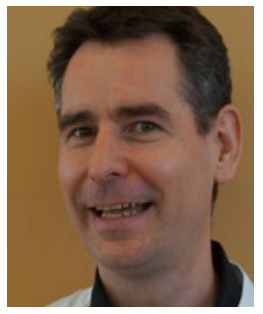

Prof. Dr. Michael Kreuter

Pneumologie und Beatmungsmedizin, Zentrum für interstitielle und seltene Lungenerkrankungen, Thoraxklinik, Universitätsklinikum Heidelberg sowie Zentrum für Translationale Lungenforschung, Heidelberg (TLRC), Mitglied des Deutschen Zentrums für Lungenforschung (DZL), Röntgenstr. 1, 69126 Heidelberg, Email: kreuter@uni-heidelberg.de

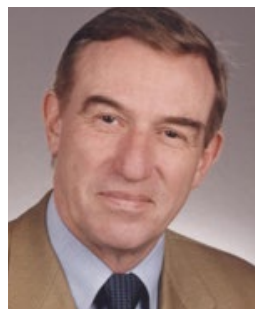

Prof. Dr. Robert Loddenkemper

Deutsche Gesellschaft für Pneumologie und Beatmungsmedizin, Robert-Koch-Platz 8, 10115 Berlin, rloddenkemper@dgpberlin.de 